\title{
Correspondence
}

\section{On a Simple Method for Detecting Synchronization Errors in Coded Messages}

\author{
Stavros Konstantinidis, Member, IEEE, Steven Perron, and \\ L. Amber Wilcox-O'Hearn
}

\begin{abstract}
We investigate the problem of designing pairs $(p, s)$ of words with the property that, if each word of a coded message is prefixed by $p$ and suffixed by $s$, the resulting set of coded messages is error detecting with finite delay. We consider (combinatorial) channels permitting any combination of the substitution, insertion, and deletion (SID) error types, and address the cases of both scattered and burst errors. A pair $(p, s)$ with the above property is evaluated in terms of three parameters: redundancy, delay of decoding, and frequency of the detectable errors. In the case of SID channels with burst errors, we provide a complete and explicit characterization of their error-detecting pairs $(p, s)$, which involves the period of the word $s p$.
\end{abstract}

Index Terms-Burst errors, decoding delay, deletion, error detection, insertion, period of word.

\section{INTRODUCTION AND BASIC NOTATION}

We investigate the problem of designing pairs $(p, s)$ of words, called separators, with the property that, if each word of a coded message is prefixed by $p$ and suffixed by $s$, the resulting coded language (set of coded messages) is error detecting with finite delay. We consider (combinatorial) channels allowing any combination of the substitution, insertion, and deletion (SID) error types. Such channels were used by Levenshtein in [1], where the method of separators was discussed for correcting scattered SID errors in coded messages. This method was first considered by Sellers Jr. [2] for a certain SID channel and, more recently, by Ferreira et al. [3]. In the present correspondence, we use the term uniform error-detector for a pair $(p, s)$ of words satisfying the above property, and we consider the cases of both scattered and burst errors. In either case, a uniform error-detector $(p, s)$ is evaluated in terms of three parameters: the redundancy $|p|+|s|$, the delay of decoding, and the frequency of the detectable errors. In the case of burst SID errors, we provide a complete and explicit characterization of all the uniform error-detectors $(p, s)$, which involves the period of the word $s p$ [4], [5].

\section{A. Notation About Alphabet, Words, and Coded Languages}

We assume an alphabet $X$ containing at least the two symbols 0 and 1. A word, or message, is any string of symbols from $X$ including the empty word $\lambda$. For a word $w$, we denote by $|w|$ the length of $w$. For example, $|11001|=5$. If $i=1, \ldots,|w|$, then $w[i]$ denotes the $i$ th symbol of $w$ and, for $j=i, \ldots,|w|$, the notation $w[i \cdots j]$ represents the word $w[i] w[i+1] \cdots w[j]$. If $j<i$, we agree that $w[i \cdots j]=\lambda$.

Manuscript received December 2, 2001; revised November 29, 2002. This work was supported by the Natural Sciences and Engineering Research Council of Canada under Grant R220259.

S. Konstantinidis and S. Perron are with the Department of Mathematics and Computing Science, Saint Mary's University, Halifax, NS B3H 3C3, Canada (e-mail: s.konstantinidis@stmarys.ca; steven_perron@hotmail.com).

L. A. Wilcox-O'Hearn is with the Department of Computer Science, University of Toronto, Toronto, ON M5S 3G4, Canada (e-mail: amber@cs.toronto. edu).

Communicated by C. Carlet, Associate Editor for Coding Theory.

Digital Object Identifier 10.1109/TIT.2003.810665
For two words $w_{1}$ and $w_{2}$ the word $w_{1} w_{2}$ is the concatenation of $w_{1}$ and $w_{2}$. For a word $w$ and a nonnegative integer $n, w^{n}$ denotes the word that consists of $n$ concatenated copies of $w$. A word $p$ is a prefix of $w$ if $w=p s$, for some word $s$. In this case, $s$ is a suffix of $w$. If $p \neq w$, then $p$ is called a proper prefix of $w$. Similarly, the suffix $s$ is proper if $s \neq w$. A word $v$ is a factor of $w$ if $w$ can be written as $x v y$ for some words $x$ and $y$. The set of all words is denoted by $X^{*}$ and the set of all nonempty words by $X^{+}$. Every subset of $X^{*}$ is called a language. If $u, v$ are words and $L$ is a language, then $u L v$ is the language $\{u w v \mid w \in L\}$. If $F$ is a finite and nonempty language, then $\ell_{F}$ denotes the maximum length of the words in $F$. For two languages $L$ and $L^{\prime}, L L^{\prime}$ denotes the language $\left\{w w^{\prime} \mid w \in L, w^{\prime} \in L^{\prime}\right\}$. If $n$ is a nonnegative integer, then $L^{n}$ is the language $\left\{w_{1} \cdots w_{n} \mid w_{1}, \ldots, w_{n} \in L\right\}$, with $L^{0}=\{\lambda\}$. Moreover, $L^{*}=\bigcup_{i=0}^{\infty} L^{i}$.

A nonempty language $C$ is called a uniquely decodable code or simply a code if, for all positive integers $m$ and $n$ and for all words

the equation

$$
v_{1}, \ldots, v_{n}, u_{1}, \ldots, u_{m} \in C
$$

$$
v_{1} v_{2} \cdots v_{n}=u_{1} u_{2} \cdots u_{m}
$$

implies $m=n$ and $u_{i}=v_{i}$ for every $i=1, \ldots, n$. In this correspondence, we assume that every code contains at least two words. If all the words of a language $C$ have the same length, then $C$ is a code and is called a uniform code. A language of the form $C^{*}$, where $C$ is a code, is called a coded language.

\section{B. Structure of the Correspondence}

This correspondence is organized as follows. In Section II, we give the basic terminology about (combinatorial) channels and error detection, and we define SID error types, error specifications, and the particular class of SID channels that permit burst errors. Moreover, we obtain a few technical results pertaining to these concepts. In Section III, we define the method of uniform error-detector pairs, discuss the criteria for choosing good pairs, and provide a necessary condition on the structure of such pairs. In Section IV, we focus on channels with burst errors and identify all uniform error-detectors for such channels, including all the optimal ones. In Section V, we consider channels with scattered errors and obtain a set of uniform error-detectors that work for any SID error type. Then, for certain error types, we identify uniform error-detectors with smaller redundancy at the cost of restricting to messages over the binary alphabet. Finally, Section VI contains a few concluding remarks.

\section{II. (COMBINATORIAL) CHANNELS AND ERROR DETECTION}

\section{A. Channels, Error Types, and Error Specifications}

A (combinatorial) channel (over the alphabet $X$ ) is a binary relation $\gamma \subseteq X^{*} \times X^{*}$. For the elements of the channel $\gamma$ we prefer to write $(z \leftarrow w)$ as opposed to $(z, w)$. Then, $(z \leftarrow w) \in \gamma$ means that the message (word) $z$ can be received from $w$ through the channel $\gamma$. Note that, in general, the channel is noisy, meaning that, for $(z \leftarrow w) \in \gamma$, it is possible that $z \neq w$; that is, $z$ is received from $w$ with errors.

In this work, we consider channels that permit combinations of the three basic error types: substitution, insertion, and deletion, denoted by the symbols $\sigma, \iota$, and $\delta$, respectively. The set of error types is

$$
\{\sigma, \iota, \delta,(\sigma \odot \iota),(\sigma \odot \delta),(\iota \odot \delta),(\sigma \odot \iota \odot \delta)\}
$$


where the symbol $\odot$ is a logic connective and is used to indicate which combination of the basic error types is permitted. The error types $\iota$ and $\delta$ are called synchronization error types. Each error type $\tau$ can be used to define a distance function $D_{\tau}$. In particular, $D_{\sigma}$ is the Hamming distance and, for $\tau \in\{(\iota \odot \delta),(\sigma \odot \iota \odot \delta)\}, D_{\tau}$ is the Levenshtein distance for errors of type $\tau$ [6].

Definition 1: An error specification is an expression of the form $m \boldsymbol{x} \tau$ such that $m$ is a nonnegative integer, $\tau$ is an error type, and $\boldsymbol{x}$ is one of the symbols $\boldsymbol{b}$ and $\boldsymbol{s}$ indicating the terms burst and scattered, respectively. For an error specification $m \boldsymbol{x} \tau$ we shall assume that $m>0$, unless stated otherwise.

Intuitively, the expression $m \boldsymbol{x} \tau$ specifies possible changes (errors) that one can make in a word to obtain another word. For example, the expression $3 \boldsymbol{b} \delta$ specifies a size 3 burst of deletion errors and the expression $4 \boldsymbol{s}(\iota \odot \delta)$ specifies four scattered insertion and deletion errors. More specifically, let $x$ and $y$ be two words. We say that $y$ obtains from $x$ using $m \boldsymbol{s} \tau$, if it is possible to transform $x$ to $y$ using exactly $m$ (scattered) errors of type $\tau$. We say that $y$ obtains from $x$ using at most $m \boldsymbol{s} \tau$, if $y$ obtains from $x$ using $k \boldsymbol{s} \tau$, for some integer $k$ with $0 \leq k \leq m$. We say that $y$ obtains from $x$ using (at most) $m \boldsymbol{b} \tau$, if there are words $p, s, u, u^{\prime}$ such that $|u| \leq m, x=p u s, y=p u^{\prime} s$, and $u^{\prime}$ obtains from $u$ using at most $m \boldsymbol{s} \tau$. For example, the word $111111 \mathrm{ob}-$ tains from 0111110 using $2 \boldsymbol{s}(\sigma \odot \delta)$, and the word 00010100 obtains from 0011100 using $2 \boldsymbol{b}(\sigma \odot \iota)$.

For an error type $\tau$ and two integers $m$ and $\ell$, with $1 \leq m<\ell$, the expression $\tau_{\boldsymbol{s}}(m, \ell)$ denotes an SID channel with scattered errors. Specifically, $(z \leftarrow w)$ is in the channel if it is possible to obtain $z$ from $w$ using errors of type $\tau$ such that no more than $m$ errors can be used in any factor of length $\ell$ (or less) of $w$. We note that channels $\tau_{\boldsymbol{s}}(m, \ell)$ with $\tau \in\{\iota, \delta,(\iota \odot \delta),(\sigma \odot \iota \odot \delta)\}$ are considered in [1] in the context of error-correcting codes for such channels. As an example, consider the channel $(\sigma \odot \delta)_{\boldsymbol{s}}(2,5)$ that permits a total of up to two substitutions and deletions in any factor of length 5 of the message. As 101000 obtains from $w=0000000$ by deleting $w[7]$ and substituting $w[3]$ with 1 and $w[1]$ with 1 , it follows that $(101000 \leftarrow$ $w) \in(\sigma \odot \delta)_{s}(2,5)$. On the other hand, to obtain 101001 from $w$ using errors of type $(\sigma \odot \delta)$, one symbol of $w$ must be deleted and three of its symbols must be substituted. But it is not possible to choose four such symbols, unless three of them occur in a factor of $w$ of length 5 . Hence, $(101001 \leftarrow w)$ is not in the channel $(\sigma \odot \delta)_{s}(2,5)$.

\section{B. Channels With Burst Errors}

Let $m$ be a positive integer. A set $B$ of $m$-burst errors is a set that consists of pairs $\left(u^{\prime} \leftarrow u\right)$ such that $|u| \leq m$. Of particular interest are the SID sets of $m$-burst errors: For any error type $\tau$, define

$B_{m}(\tau)=\left\{\left(u^{\prime} \leftarrow u\right)|| u \mid \leq m\right.$ and $u^{\prime}$ obtains from $u$ using

at most $m \boldsymbol{b} \tau$ \}.

Given a set $B$ of $m$-burst errors and an integer $\ell>m$, we define the channel $\gamma_{\ell}[B]$ as follows: $(z \leftarrow w)$ is in $\gamma_{\ell}[B]$ if and only if there is an integer $n \geq 0$ and words $x_{0}, \ldots, x_{n}, u_{1}, \ldots, u_{n}, u_{1}^{\prime}, \ldots, u_{n}^{\prime}$ such that

- $w=x_{0} u_{1} x_{1} \cdots u_{n} x_{n}$ and $z=x_{0} u_{1}^{\prime} x_{1} \cdots u_{n}^{\prime} x_{n}$;

- for all $i=1, \ldots, n-1,\left|x_{i}\right| \geq \ell-1$;

- $\left(u_{i}^{\prime} \leftarrow u_{i}\right)$ is in $B$ and $u_{i}^{\prime} \neq u_{i}$, for all $i=1, \ldots, n$.

Informally, the above conditions mean that if $z$ obtains from $w$ through $\gamma_{\ell}[B]$ then, in $w$, there are zero or more bursts of errors each of size $m$ (or less) such that there is at most one burst (or part of a burst) in any $\ell$ consecutive symbols of $w$. In case $B=B_{m}(\tau)$, we call the channel $\gamma_{\ell}\left[B_{m}(\tau)\right]$ an SID channel with burst errors and we shall use the notation $\tau_{\boldsymbol{b}}(m, \ell)$ instead of $\gamma_{\ell}\left[B_{m}(\tau)\right]$ which is consistent with the notation we use for SID channels with scattered errors. For example, let $w=0^{2} 110^{7} 110^{8} 1$ and let $z=0^{2} 1000^{7} 00^{8} 010$. Then $(z \leftarrow w)$ is in the channel $(\sigma \odot \iota \odot \delta)_{\boldsymbol{b}}(2,8)$.

\section{Error Detection With Finite Delay}

Let $\gamma$ be a channel. A language $L$ is called error detecting for $\gamma$ when the following condition is satisfied: Assuming only words in $L \cup\{\lambda\}$ are sent into $\gamma$, if a word $y$ is received through $\gamma$ and $y$ is in $L \cup\{\lambda\}$ then $y$ must be correct; that is, equal to the transmitted word. This condition can be written more formally as follows [7]:

$$
\begin{gathered}
\text { For all words } x \in L \cup\{\lambda\} \text { and } y \in X^{*}, \\
\text { if }(y \leftarrow x) \in \gamma \text { and } y \in L \cup\{\lambda\} \text {, then } y=x .
\end{gathered}
$$

Note that the above condition ensures that a nonempty word of $L$ cannot be received from the empty word, and the empty word cannot be received from a nonempty word of $L$. The task of verifying that a language is error detecting could require some effort even for apparently simple languages and channels. We invite the reader to show that, for $K=\{1011,1101\}$, the coded language $K^{*}$ is error detecting for the SID channel $\delta_{\boldsymbol{b}}(1,4)$ that allows up to one deletion in any four consecutive symbols of a message.

Although error detection is a basic property of a communications language, the process of decoding a word of such a language might require unbounded memory. This is because, in general, the decoder needs to see the entire message in order to decide whether it is correct. For coded languages, however, it is possible to define the concept of error detection with finite delay as follows. If a codeword $v$ is observed at the beginning of the received message, then $v$ is correct - that is, equal to the first codeword of the transmitted message-provided that there are at least $d$ codewords following $v$ in the received message. Moreover, once $v$ is decoded, the rest of the received message can be decoded in the same way. The number $d$ is the delay of decoding. In case the received message does not begin with $1+d$ codewords, an error is detected and what follows depends on the communication protocol-usually involving retransmission techniques. More formally, for a channel $\gamma$ and a nonnegative integer $d$, we say that the coded language $C^{*}$ is error detecting for $\gamma$ with delay $d$, when the following condition is satisfied for all $v \in C, z \in C^{d} X^{*}$, and $w \in C^{*}$ :

if $(v z \leftarrow w) \in \gamma$, then $w=v u$ and $(z \leftarrow u) \in \gamma$ for some $u \in C^{*}$.

For any reasonable channel $\gamma$-an SID channel, for instance-if $C^{*}$ satisfies the above condition then $C^{*}$ is indeed error detecting for $\gamma$ [7]. Moreover, if $C^{*}$ satisfies the above then the code $C$ has finite decoding (deciphering) delay at most $d$ (in the sense of [8]).

Example 1: Consider again the code $K=\{1101,1011\}$ and recall that the language $K^{*}$ is error detecting for $\delta_{\boldsymbol{b}}(1,4)$. However, $K^{*}$ is not error detecting for that channel with any finite delay. Indeed, assume that $K^{*}$ is error detecting with delay $d$, for some nonnegative integer $d$, and consider the words $w=(1101)^{d+2}, v=1011$, and $z=(1011)^{d} 101$. By deleting the first 1 of $w$ we have that $(v z \leftarrow w)$ is in the channel which implies that $w$ must start with the codeword $v$; a contradiction.

Example 2: Consider the code $C=\{001,011\}$ and the channel $\delta_{\boldsymbol{b}}(1,4)$. Then, $C^{*}$ is error detecting for $\delta_{\boldsymbol{b}}(1,4)$ with delay 0 . Indeed, suppose $v \in C, z \in X^{*}$, and $w \in C^{*}$ such that $(v z \leftarrow w)$ is in $\delta_{\boldsymbol{b}}(1,4)$. Note that $w$ must start with a codeword of the form $0 b 1$ for some alphabet symbol $b$. Then, $w=0 b 1 u$ for some $u \in C^{*}$. Suppose that $0 b 1$ results in some word $z_{1}$ and $u$ results in some word $z_{2}$ such that $v z=z_{1} z_{2}$. If there is a deletion in $0 b 1$, then $|v|=\left|z_{1}\right|+1$ and there can be no error on the symbol $u[1]$ that follows $0 b 1$. As $u[1]=0$, we have that $v=z_{1} 0$, which contradicts the assumption $v \in C$. Thus, there can be no deletion in $01 b$, which implies that $v=z_{1}=0 b 1$ and $z_{2}=z$ and, therefore, $(z \leftarrow u) \in \delta_{\boldsymbol{b}}(1,4)$. The code $C$ allows 
for the correct decoding of messages in $C^{*}$ without delay as long as no error occurs. For example, suppose that the concatenation of codewords $001,011,001,001, \ldots$ is transmitted and a deletion occurs in the third codeword, 001 , so that we receive $001011010 \cdots$. Then, the codewords 001 and 011 can be decoded correctly and an error is detected when 010 is observed.

\section{Some Technical Results}

We close this section with a few technical results concerning error specifications and SID channels, which are needed in the sequel. The proofs can be found in the Appendix.

Lemma 1: Let $m \boldsymbol{x} \tau$ be an error specification, other than $m \boldsymbol{s}(\iota \odot \delta)$ and $m \boldsymbol{s}(\sigma \odot \iota \odot \delta)$, containing at least two different error types and let $v$ and $v^{\prime}$ be two words of the same length. Then, $v^{\prime}$ obtains from $v$ using at most $m \boldsymbol{x} \tau$ if and only if $v^{\prime}$ obtains from $v$ using at most $m \boldsymbol{x} \sigma$.

Lemma 2: Let $m \boldsymbol{x} \tau$ be an error specification and let $p, v, v^{\prime}, s$ be words such that $v$ and $v^{\prime}$ are of the same length. Then, $p v^{\prime} s$ obtains from pvs using at most $m \boldsymbol{x} \tau$ if and only if $v^{\prime}$ obtains from $v$ using at most $m \boldsymbol{x} \tau$.

Next we establish certain relationships between sets of the form $B_{m}(\tau)$ involving different error types.

Lemma 3: Let $m$ be a positive integer and let $u$ and $u^{\prime}$ be two words such that $|u| \leq m$ and $u^{\prime}$ obtains from $u$ using at most $m \boldsymbol{s}(\sigma \odot \iota \odot \delta)$.

1) If $\left|u^{\prime}\right| \leq|u|$, then $u^{\prime}$ obtains from $u$ using at most $m \boldsymbol{s}(\sigma \odot \delta)$.

2) If $\left|u^{\prime}\right|>|u|$, then $u^{\prime}$ obtains from $u$ using at $\operatorname{most} \max \{1,2 m-$ $2\} \boldsymbol{s}(\sigma \odot \iota)$.

3) If the alphabet $X$ is binary and $\left|u^{\prime}\right|=|u|+1$, then $u^{\prime}$ obtains from $u$ using at most $m \boldsymbol{s}(\sigma \odot \iota)$.

Proposition 1: For all integers $m \geq 2$

$$
B_{m}(\sigma \odot \iota \odot \delta) \subseteq B_{m}(\sigma \odot \delta) \cup B_{2 m-2}(\sigma \odot \iota) .
$$

Proof: The claim follows easily from the previous lemma.

A natural question that arises is whether Proposition 1 can be strengthened by replacing $B_{2 m-2}(\sigma \odot \iota)$ with $B_{m}(\sigma \odot \iota)$. It turns out that this is possible only when $m<5$.

Proposition 2: Consider the alphabet $X$ and a positive integer $m$. Then, $B_{m}(\sigma \odot \iota \odot \delta)=B_{m}(\sigma \odot \delta) \cup B_{m}(\sigma \odot \iota)$ if and only if $m \in\{1,2\}$, or $m \in\{3,4\}$ and $X=\{0,1\}$.

The above considerations motivate us to define a new type of SID channels with burst errors as follows. For two error types $\tau_{1}$ and $\tau_{2}$, let $\left(\tau_{1} \vee \tau_{2}\right)_{\boldsymbol{b}}(m, \ell)$ be the channel $\gamma_{\ell}\left[B_{m}\left(\tau_{1}\right) \cup B_{m}\left(\tau_{2}\right)\right]$. Then, every burst of errors in a transmitted message is of type $m \boldsymbol{b} \tau_{1}$ or $m \boldsymbol{b} \tau_{2}$. By Proposition 2, it follows that, for $X=\{0,1\}$ and $m<5$, the channel $((\sigma \odot \iota) \vee(\sigma \odot \delta))_{\boldsymbol{b}}(m, \ell)$ coincides with the channel $(\sigma \odot \iota \odot \delta)_{\boldsymbol{b}}(m, \ell)$.

\section{The Method of SEPARATORS FOR DETECTING SID ERRORS}

In [1], Levenshtein briefly discusses the method of separators for correcting certain scattered SID errors in messages, with finite delay. Loosely speaking, if a coded language $K^{*}$ is error correcting for a channel $\gamma$ with finite delay then, for every received message $w$, it is possible to determine the first codeword of the original message by looking only at a prefix of $w$ of bounded length. The method of separators involves choosing an appropriate pair of words $(p, s)$ such that $(p C s)^{*}$ is error correcting for $\gamma$ with finite delay, for any uniform code $C$ that is error correcting for $\gamma$. In this section, we use the above idea to define a formal method for obtaining coded languages of the form $(p C s)^{*}$ that are error detecting with finite delay for SID channels with scattered errors or with burst errors.

Let $m$ be a positive integer. The symbol $\mathcal{U}^{m}$ denotes the class of all uniform codes of length greater than $m$. For an error specification $m \boldsymbol{x} \tau$, we write $\mathcal{U}_{m \boldsymbol{x} \tau}$ for the class of all uniform codes $C$ such that $C$ is error detecting for $\tau_{\boldsymbol{x}}\left(m, \ell_{C}\right)$-recall, $\ell_{C}$ is the word length of the code $C$. By the definition of error detection and by Lemma 1, the following obtains.

Lemma 4: Let $m \boldsymbol{x} \tau$ be an error specification.

- If $\tau \in\{\iota, \delta\}$, then $\mathcal{U}_{m \boldsymbol{x} \tau}=\mathcal{U}^{m}$.

- If $\tau \notin\{\iota, \delta\}$ and $\boldsymbol{x}=\boldsymbol{b}$, then $\mathcal{U}_{m \boldsymbol{x} \tau}=\mathcal{U}_{m \boldsymbol{b} \sigma}$.

- If $\tau \in\{\sigma,(\sigma \odot \delta),(\sigma \odot \iota)\}$ and $\boldsymbol{x}=\boldsymbol{s}$, then $\mathcal{U}_{m \boldsymbol{x} \tau}=\mathcal{U}_{m \boldsymbol{s} \sigma}$.

It follows also that

$$
\mathcal{U}_{m \boldsymbol{s}(\sigma \odot \ell \odot \delta)} \subseteq \mathcal{U}_{m \boldsymbol{s} \sigma} \subseteq \mathcal{U}_{m \boldsymbol{b} \sigma} \subseteq \mathcal{U}^{m}
$$

Codes in the classes $\mathcal{U}_{m \boldsymbol{s} \sigma}$ and $\mathcal{U}_{m \boldsymbol{b} \sigma}$ have been studied extensivelysee [9], for instance. On the other hand, at a first glance, it appears that codes in the classes $\mathcal{U}_{m \boldsymbol{s}(\sigma \odot \iota \odot \delta)}$ and $\mathcal{U}_{m \boldsymbol{s}(\iota \odot \delta)}$ have not been considered in the past. By the results of [1], however, the following obtains.

Remark 1: Let $\tau \in\{(\iota \odot \delta),(\sigma \odot \iota \odot \delta)\}$ and let $m$ be a positive integer. A uniform code $C$ is error correcting for $\tau_{\boldsymbol{s}}\left(m, \ell_{C}\right)$ if and only if it is error detecting for $\tau_{\boldsymbol{s}}\left(2 \mathrm{~m}, \ell_{C}\right)$.

The above follows when we note that i) a code $C$ is error correcting for $\tau_{\boldsymbol{s}}\left(m, \ell_{C}\right)$ if and only if $D_{\tau}(C)>2 m$, and ii) a code $C$ is error detecting for $\tau_{\boldsymbol{s}}\left(m, \ell_{C}\right)$ if and only if $D_{\tau}(C)>m$. We also note that, to our knowledge, only very few general construction methods exist for codes that are error correcting for $(\iota \odot \delta)_{\boldsymbol{s}}\left(m, \ell_{C}\right)$-see, for instance, [10]-[12].

Definition 2: Let $m \boldsymbol{x} \tau$ be an error specification. A pair of words $(p, s)$ is called a uniform error-detector for $m \boldsymbol{x} \tau$ (or simply an $m \boldsymbol{x} \tau$ detector) if there are two nonnegative integers $d$ and $t$ such that, for every code $C$ in $\mathcal{U}_{m \boldsymbol{x} \tau}$, the coded language $(p C s)^{*}$ is error detecting for $\tau_{\boldsymbol{x}}\left(m, \ell_{C}+|p s|+t\right)$ with delay $d$. Then, we say that $(p, s)$ has redundancy $|p|+|s|$, delay $d$, and offset $t$.

For a given error specification $m \boldsymbol{x} \tau$, the design of a uniform $m \boldsymbol{x} \tau$-detector should consider the following criteria.

1) Low redundancy of the encoding $C \mapsto p C s$ : This is achieved by choosing a pair $(p, s)$ with small redundancy $|p|+|s|$.

2) High frequency of the errors detectable by $(p C s)^{*}$ : This is achieved by choosing a pair $(p, s)$ with small offset $t$. Indeed, an $m \boldsymbol{x} \tau$-detector $(p, s)$ with offset $t$ ensures the detection of $m$ errors of type $\tau$ in any $\ell_{C}+|p s|+t$ symbols of the transmitted message. Thus, the smaller is the value of $t$ the higher is the ratio $m /\left(\ell_{C}+|p s|+t\right)$.

3) Small amount of memory for decoding words in $(p C s)^{*}$ : This is achieved by choosing a pair $(p, s)$ with small delay $d$.

Our primary criterion will be the optimization of the redundancy of a uniform error-detector $(p, s)$. With this constraint, we shall attempt to define error-detectors with minimal delay and minimal offset.

The first result gives a necessary condition on the structure of uniform error-detectors that involves the notion of period of a word. A positive integer $k$ is called a period of a nonempty word $w$, if $w[i]=$ $w[k+i]$ for every $i \in\{j \mid 1 \leq j$ and $j \leq|w|-k\}$-note that this condition is vacuously true when $k \geq|w|$ and, in this case, the number $k$ is a period of $w$. The smallest $k$ satisfying this condition is called the period of the word $w$ and we denote it by $\operatorname{per}(w)$. It should be clear that $1 \leq \operatorname{per}(w) \leq|w|$. This concept is important in various domains 
including pattern matching algorithms and game theory, [13], and word combinatorics [4].

\section{Lemma 5:}

1) For any nonempty word $w$ there are words $u, x, y$ such that $w=u x=y u$ and $|x|=|y|=\operatorname{per}(w)$.

2) For every nonempty words $u_{1}, x_{1}, u_{2}, x_{2}$ with $\left|u_{1}\right|=\left|u_{2}\right|$ and $u_{1} x_{1}=x_{2} u_{2}$, if $\operatorname{per}\left(u_{1} x_{1}\right)>\left|x_{1}\right|$ then $u_{1} \neq u_{2}$

Proof: The statements follow easily if we note that $k$ is a period of $w$ if and only if, either $k \geq|w|$ or $w=x u=u y$ for some $u \in X^{+}$ and $x, y \in X^{k}$.

Proposition 3: Let $m \boldsymbol{x} \tau$ be an error specification not in $\{m \boldsymbol{x} \sigma, m \boldsymbol{s}(\sigma \odot \iota \odot \delta), m \boldsymbol{s}(\iota \odot \delta)\}$. If a pair of words $(p, s)$ is a uniform error-detector for $m \boldsymbol{x} \tau$, then $\operatorname{per}(s p)>m$ and, therefore, the redundancy of $(p, s)$ is greater than $m$.

Proof: Assume $(p, s)$ is a uniform $m \boldsymbol{x} \tau$-detector and consider first the case where $|s p|>0$. Then, $s p=u x=y u$ for some words $u, x, y$ with $|x|=|y|=\operatorname{per}(s p)$. Suppose $\operatorname{per}(s p) \leq m$. We shall obtain a contradiction by constructing a uniform code $C$ that is error detecting for the channel $\sigma_{s}\left(m, \ell_{C}\right)$ but the coded language $(p C s)^{*}$ is not error detecting for $\tau_{x}\left(m, \ell_{C}+|p s|+t\right)$ with finite delay, for any $\boldsymbol{x} \in\{\boldsymbol{b}, \boldsymbol{s}\}$ and for any nonnegative integer $t$. Let $v=\left(1^{|x|} 0^{|x|}\right)^{1+\lceil m /(2|x|)\rceil}$. Then, the words $x v$ and $v y$ are of the same length and they differ in at least $m+1$ positions; therefore, the code $C=\{x v, v y\}$ is error detecting for $\sigma_{s}\left(m, \ell_{C}\right)$. Now assume $(p C s)^{*}$ is error detecting for $\tau_{\boldsymbol{x}}\left(m, \ell_{C}+|p s|+t\right)$ with delay $d$, for some nonnegative integers $t$ and $d$. Let $w$ be the word $(\text { pxvs })^{d+3}=$ pxvyuxv $(y u x v)^{d+1} s$ if $\tau$ contains $\delta$, or $(\text { pvys })^{d+2}$ otherwise. Consider also the word

$$
z= \begin{cases}\operatorname{pxvuxv}(y u x v)^{d+1} s, & \text { if } \tau \text { contains } \delta \\ \text { pxvys }(\text { pvys })^{d+1}, & \text { otherwise. }\end{cases}
$$

In the first case, $z$ obtains from $w$ by deleting the word $y$. In the second case, $z$ obtains from $w$ by inserting the word $x$. Moreover, as the word $z$ can be written as

$$
\begin{cases}(\text { pxvs })(p v y s)(p v y s)^{d} p v s, & \text { if } \tau \text { contains } \delta \\ \text { pxvs }(p x v s)^{d} \text { pxvys, } & \text { otherwise }\end{cases}
$$

the assumption about $(p C s)^{*}$ implies that $p x v s=$ pvys which in turn implies that $x v=v y$; a contradiction.

Finally, consider the case where $|s p|=0$. Define the code $C=$ $\left\{0^{m} 1^{m}, 1^{m} 0^{m}\right\}$ which is error detecting for the channel $\sigma_{\boldsymbol{s}}\left(m, \ell_{C}\right)$. Then, depending on $\tau$, one can choose words $y$ and $u$ such that

$$
\left(0^{m} 1^{m}\left(0^{m} 1^{m}\right)^{d} y \leftarrow\left(1^{m} 0^{m}\right)^{d+1} u\right) \in \tau_{\boldsymbol{x}}\left(m, \ell_{C}\right)
$$

for any nonnegative integer $d$. Hence, $C^{*}$ is not error detecting for $\tau_{\boldsymbol{x}}\left(m, \ell_{C}+t\right)$ with finite delay, for any offset $t$.

\section{THE CASE OF Burst ERRORS}

In this section, we provide a detailed analysis on the structure of uniform error-detectors for SID channels with burst errors. The analysis allows us to identify all such error-detectors, including all the ones that are optimal in terms of redundancy, delay, and offset.

Proposition 4: Let $m \boldsymbol{b} \tau$ be a burst-error specification and let $(p, s)$ be a pair of words with $|s p|>0$. If $\operatorname{per}(s p)>m$, then $(p, s)$ is a uniform error-detector for $m \boldsymbol{b} \tau$ with offset 1 and delay 2 . Moreover, if $p$ is empty then $(p, s)$ has delay 1 .

Proof: Suppose $\operatorname{per}(s p)>m$ and let $C$ be any code in $\mathcal{U}_{m b \sigma}$. We show that $(p C s)^{*}$ is error detecting for $\gamma=\tau_{\boldsymbol{b}}\left(m, \ell_{C}+|s p|+1\right)$ with delay $d \in\{1,2\}$, where $d=1$ if $|p|=0$. For this, assume $\left(p v_{0} s \cdots p v_{d} s y \leftarrow p w_{0} s p w_{1} s u\right) \in \gamma$ where $w_{0}, w_{1}, v_{0}, \ldots, v_{d} \in C$, $y \in X^{*}$ and $u \in(p C s)^{*}$. We need to show that $v_{0}=w_{0}$ and $\left(p v_{1} s \cdots p v_{d} s y \leftarrow p w_{1} s u\right) \in \gamma$. If there are no errors in $p w_{0} s$, then we are done. So assume there is a burst $\left(x^{\prime} \leftarrow x\right) \in B_{m}(\tau)$, with $x \neq x^{\prime}$, that affects $p w_{0} s$; that is, there are words $z_{1}, z_{2}, z_{3}, z_{3}^{\prime}$ with $\left|z_{1}\right|<\left|p w_{0} s\right|$ and $\left|z_{2}\right| \geq \ell_{C}+|s p|$ such that $p w_{0} s p w_{1} s u=z_{1} x z_{2} z_{3}$ and $p v_{0} s \cdots p v_{d} s y=z_{1} x^{\prime} z_{2} z_{3}^{\prime}$ and $\left(z_{3}^{\prime} \leftarrow z_{3}\right) \in \gamma$. Obviously, this is the only burst that affects $p w_{0} s$. Let $q=\left|x^{\prime}\right|-|x|$. Then, $0 \leq|q| \leq m$. We distinguish six cases about the sign of $q$ and the position of the burst $\left(x^{\prime} \leftarrow x\right)$ in $p w_{0} s p w_{1} s u$.

Case 1: $q=0$ and the burst occurs before the factor $w_{1}$ of $p w_{0} s p w_{1} s u$; that is, $\left|z_{1} x\right| \leq\left|p w_{0} s p\right|$ and $p w_{0} s p$ results in $p v_{0} s p$, which implies that $v_{0}=w_{0}$, as $p C s p$ is in $\mathcal{U}_{m \boldsymbol{b} \tau}$, and

$$
\left(v_{1} s \cdots p v_{d} s y \leftarrow w_{1} s u\right) \in \gamma .
$$

Hence, also $\left(p v_{1} s \cdots p v_{d} s y \leftarrow p w_{1} s u\right) \in \gamma$, as required.

Case 2: $q=0$ and the burst affects the factor $w_{1}$ of $p w_{0} s p w_{1} s u$; that is, $\left|z_{1} x\right|>\left|p w_{0} s p\right|$. Moreover, as $|x| \leq m$ and $\left|z_{1}\right|<\left|p w_{0} s\right|$ and $|s p|>m$, we have that $x$ is of the form $s_{2} p x_{1}$ and $x^{\prime}$ is of the form $s_{2} p y_{1}$, for some prefix $x_{1}$ of $w_{1}$ and for some prefix $y_{1}$ of $v_{1}$ and for some suffix $s_{2}$ of $s$. Now as there are no errors in $z_{2}$, it follows that the codewords $v_{1}$ and $w_{1}$ differ in at most $\left|x_{1}\right|$ symbols. Hence, $w_{1}=v_{1}$ which implies that $x_{1}=y_{1}$. This, however, contradicts the fact that $x \neq x^{\prime}$.

In the next four cases we assume $|q| \neq 0$. Then, $s p=x_{1} u_{1}=u_{2} x_{2}$ for some words $x_{1}, x_{2}, u_{1}, u_{2}$ such that $\left|x_{1}\right|=\left|x_{2}\right|=|q|$ and $\left|u_{1}\right|=$ $\left|u_{2}\right|=|s p|-|q|$. As $\operatorname{per}(s p)>|q|$, it follows that $u_{1} \neq u_{2}$. Let $k$ be the largest position of $u_{1}$ and $u_{2}$ such that $u_{1}[k] \neq u_{2}[k]$.

Case 3: $q<0$ and the burst occurs before the position $|q|+k$ of the factor $s p=x_{1} u_{1}$ of $p w_{0} s p w_{1} s u$; that is,

$$
\left|z_{1} x\right| \leq\left|p w_{0} x_{1} u_{1}[1 \cdots k-1]\right| \text {. }
$$

In this case, $p w_{0} x_{1} u_{1}[1 \cdots k-1]$ results in the prefix $p v_{0} u_{2}[1 \cdots k-1]$ of $p v_{0} s \cdots p v_{d} s y$ and the next symbol $u_{1}[k]$ results in $u_{2}[k]$ with no errors. This contradicts the fact that $u_{1}[k] \neq u_{2}[k]$.

Case 4: $q<0$ and the burst contains the position $|q|+k$ of the factor $s p=x_{1} u_{1}$ of $p w_{0} s p w_{1} s u$; that is,

$$
\left|z_{1} x\right| \geq\left|p w_{0} x_{1} u_{1}[1 \cdots k]\right|
$$

In this case, there can be no error in the prefix $x_{1} u_{1}[1 \cdots k]$ of the second $s p$ in $p w_{0} s p w_{1} s p$. Then, $p w_{0} s p w_{1} x_{1}$ results in $p v_{0} s p v_{1}$ and the next $k$ symbols $u_{1}[1 \cdots k]$ result in the prefix $u_{2}[1 \cdots k]$ of $s p$ with no errors. This contradicts the fact that $u_{1}[k] \neq u_{2}[k]$.

Case 5: $q>0$ and the burst occurs before the position $k$ of $s p=$ $u_{2} x_{2}$; that is,

$$
\left|z_{1} x\right| \leq\left|p w_{0} u_{2}[1 \cdots k-1]\right| .
$$

In this case, $p w_{0} u_{2}[1 \cdots k-1]$ results in the prefix $p v_{0} x_{1} u_{1}[1 \cdots k-1]$ of $p v_{0} s \cdots p v_{d} s y$ and the next symbol $u_{2}[k]$ results in $u_{1}[k]$ with no errors. This contradicts the fact that $u_{1}[k] \neq u_{2}[k]$.

Case 6: $q>0$ and the burst contains the position $k$ of the factor $s p=u_{2} x_{2}$ of $p w_{0} s p w_{1} s u$; that is,

$$
\left|z_{1} x\right| \geq\left|p w_{0} u_{2}[1 \cdots k]\right| \text {. }
$$

In this case, there can be no error in the prefix $u_{2}[1 \cdots k]$ of the second $s p$ in $p w_{0} s p w_{1} s p$. Then, $p w_{0} s p w_{1}$ results in $p v_{0} s p v_{1} x_{1}$ and the next $k$ symbols $u_{2}[1 \cdots k]$ result in the suffix $u_{1}[1 \cdots k]$ of $s p$ with no errors. This contradicts the fact that $u_{1}[k] \neq u_{2}[k]$.

For burst-error specifications, Propositions 3 and 4 provide a complete characterization of the structure of their uniform error-detectors. Moreover, it is possible to characterize precisely all such error-detectors $(p, s)$ having optimal redundancy. Indeed, Proposition 3 implies 
that $m+1$ is the smallest value of $|p|+|s|$ and this value is possible when $\operatorname{per}(s p) \geq m+1$. But, as $\operatorname{per}(s p) \leq|s p|$, it follows that $(p, s)$ is an error-detector with optimal redundancy when $|s p|=\operatorname{per}(s p)$. This condition is equivalent to the constraint that the word $s p$ is unbordered [4] (or self-uncorrelated [14]): no proper and nonempty prefix of $s p$ is also a suffix of $s p$. It turns out that there are many unbordered words even for binary alphabets: about $27 \%$ of all binary words are unbordered, and this quantity increases for larger alphabets [13]. For example, the words $0^{7} 1$ and $0^{2} 1^{2} 0101$ are unbordered and, therefore, the pairs $\left(1,0^{7}\right)$ and $\left(1^{2} 0101,0^{2}\right)$ are uniform $7 \boldsymbol{b} \tau$-detectors with delay at most 2 and offset 1 for every error type $\tau$. We summarize the preceding remarks as follows.

Corollary 1: Let $m \boldsymbol{b} \tau$ be a burst-error specification. A pair of words $(p, s)$ is a uniform $m \boldsymbol{b} \tau$-detector with optimal redundancy if and only if $|s p|=m+1$ and the word $s p$ is unbordered.

The next result concerns the question of whether an error-detector with optimal redundancy can have offset 0 - the proof can be found in the Appendix

Proposition 5: Let $m \boldsymbol{b} \tau$ be a burst-error specification containing at least two different basic error types and such that $m \boldsymbol{b} \tau \neq 1 \boldsymbol{b}(\iota \odot \delta)$. If $(p, s)$ is an error-detector for $m \boldsymbol{b} \tau$ with redundancy $m+1$, then $(p, s)$ has offset greater than zero.

Consider an error type $\tau$ that permits insertion errors. In [7], it is shown that there exists no coded language that is error-detecting with delay 0 for any SID channel of the form $\tau_{\boldsymbol{s}}(m, \ell)$. The argument used for proving this statement can also be repeated for channels of the form $\tau_{\boldsymbol{b}}(m, \ell)$. On the other hand, for an error type $\tau$ in $\{\delta,(\sigma \odot \delta)\}$ it is possible to define $m \boldsymbol{b} \tau$-detectors with delay 0 . In fact, we obtain a precise characterization of all those error-detectors which shows that the process of choosing a pair $(p, s)$ from a word $w=s p$ is important when it comes to the delay of $(p, s)$ as an error-detector. This observation follows also from Proposition 4 , where an empty $p$ ensures that $(p, s)$ has delay 1 .

Lemma 6: Let $m \boldsymbol{b} \tau$ be a burst-error specification and let $\ell$ be an integer greater than $m$. For every $(z \leftarrow w)$ in the channel $\tau_{\mathbf{b}}(m, \ell)$ one has that

$$
|w|-|z| \leq m\lfloor|w| /(\ell-1+m)\rfloor+\min \{m,|w| \%(\ell-1+m)\}
$$

where $|w| \%(\ell-1+m)$ is the remainder of the integer division $|w| /(\ell-1+m)$.

Proposition 6: Let $\tau$ be an error type in $\{\delta,(\sigma \odot \delta)\}$. A pair of words $(p, s)$ is a uniform error-detector for $m \boldsymbol{b} \tau$ with delay 0 and offset 1 if and only if $s \in X^{*} a$ and $p \in(X \backslash\{a\})^{m} X^{*}$ for some symbol $a \in X$.

Proof: First we consider the "if" part. Assume that $s \in X^{*} a$ and $p \in(X \backslash\{a\})^{m} X^{*}$, and consider a code $C$ in $\mathcal{U}_{m \boldsymbol{b} \sigma}$ and the channel $\gamma=\tau_{\boldsymbol{b}}\left(m, \ell_{C}+|s p|+1\right)$. We show that $(p C s)^{*}$ is error detecting for $\gamma$ with delay 0 . For this, suppose $\left(p v_{0} s y \leftarrow p w_{0} s u\right) \in \gamma$, where $v_{0}, w_{0} \in C, y \in X^{*}$, and $u \in(p C s)^{*}$. Then, $p w_{0} s$ results in a prefix $z_{0}$ of $p v_{0} s y$ and $u$ results in the word $z$ with $z_{0} z=p v_{0} s y$; therefore, $(z \leftarrow u) \in \gamma$. If $\left|z_{0}\right|=\left|p w_{0} s\right|$, then $z=y$ and $z_{0}=p v_{0} s$ which implies that $(y \leftarrow u)$ is in $\gamma$ and that $p v_{0} s=p w_{0} s$ as $C$ is in $\mathcal{U}_{m \boldsymbol{b} \sigma}$. In this case, we are done. Now assume that $\left|z_{0}\right|<\left|p w_{0} s\right|$. Then a burst of errors affects $p w_{0} s$ and there are $d=\left|p w_{0} s\right|-\left|z_{0}\right|$ deletions in $p w_{0} s$. Moreover, $u=p w_{1} s u^{\prime}$ for some $w_{1}$ in $C$ and $u^{\prime} \in(p C s)^{*}$. Now $p v_{0} s$ can be written as $z_{0} z_{1}$ such that $\left|z_{1}\right|=d$ and $z_{1}$ obtains from some prefix of $p w_{1} s$. In particular, $z_{1}[d]$, the last symbol of $z_{1}$, obtains from some symbol $p[i] \in X \backslash\{a\}$ of $p$ for some $i$ in $\{1, \ldots, m\}$. Moreover, it follows that $z_{1}$ obtains from $p[1 \cdots i]$ through $\gamma$. If $\tau=\delta$, then $z_{1}[d]=p[i]$ which is impossible and the "if" part is complete for the case where $\tau=\delta$. So suppose that $\tau=(\sigma \odot \delta)$ and that $p[i]$ is substituted with $a=z_{1}[d]$.

First we argue that there is only one burst of errors in $p w_{0} s p[1 \cdots i]$. Indeed, if there are two bursts then there will be at least $\ell_{C}+|s p|$ symbols of $p w_{0} s p[1 \cdots i]$ between the bursts and, therefore, the first burst would occur in some prefix $p\left[1 \cdots i_{1}\right]$ of $p$, where $i>i_{1} \geq d$. Moreover, the second burst would occur after the prefix $p\left[1 \cdots i_{1}\right]$ of $p[1 \cdots i]$. But then the last symbol $a$ of $p v_{0} s$ would be equal to the symbol $p[d]$ of $p\left[1 \cdots i_{1}\right]$ which is impossible. Hence, only one burst of $(\sigma \odot \delta)$ errors in $p w_{0} s p[1 \cdots i]$ such that the last symbol of $p[1 \cdots i]$ is substituted with $a=z_{1}[d]$. The beginning of the burst will be at or after the position $|p|+\ell_{C}-m+2$ of $p w_{0}$ which implies that $v_{0}$ differs from $w_{0}$ in at most $m-2$ consecutive symbols. Hence, as $C$ is in $\mathcal{U}_{m \boldsymbol{b} \sigma}$, it follows that $p w_{0} s=p v_{0} s$. It remains to show that $(y \leftarrow u)$ is in $\gamma$.

Consider in more detail the first burst, say

$$
\left(u_{1}^{\prime} z_{1} u_{2}^{\prime} \leftarrow u_{1} p[1 \cdots i] u_{2}\right)
$$

that occurs in $p w_{0} s p w_{1} s u^{\prime}$ to get $z_{0} z_{1} y$, such that $p w_{0} s=x u_{1}$ for some word $x, z_{0}=x u_{1}^{\prime}, p[i+1 \cdots|p|] w_{1} s u^{\prime}=u_{2} x_{2}$ for some word $x_{2}$, and $y \in u_{2}^{\prime} X^{*}$ with $\left(y \leftarrow u_{2} x_{2}\right) \in \gamma$. Then, also $\left(y \leftarrow p[1 \cdots i] u_{2} x_{2}\right)$ is in $\gamma$ by deleting the prefix $p[1 \cdots i]$ of $p[1 \cdots i] u_{2} x_{2}$ and keeping the rest of the error bursts that existed in $u_{2} x_{2}$. Note that this is possible since the $i$ deletions required for that already existed in the part $\left(u_{1}^{\prime} z_{1} \leftarrow u_{1} p[1 \cdots i]\right)$ of the burst $\left(u_{1}^{\prime} z_{1} u_{2}^{\prime} \leftarrow u_{1} p[1 \cdots i] u_{2}\right)$-recall the $d$ deletions in $u_{1}$ and the $|p[1 \cdots i]|-\left|z_{1}\right|$ deletions in $p[1 \cdots i]$ to obtain $z_{1}$. Hence, the "if" part is complete when we recall that $u=p[1 \cdots i] u_{2} x_{2}$.

We turn now to the "only if" part. Assume that $(p, s)$ is a uniform error-detector for $m \boldsymbol{b} \tau$ with delay 0 and offset 1 . Then, $|s p|>m$ by Proposition 3. Suppose it is not the case that $s \in X^{*} a$ and $p \in$ $(X \backslash\{a\})^{m} X^{*}$. We distinguish three cases. In the first case, $s=\lambda$. Let $y=p[1 \cdots m]$ and let $C=\{y z, z y\}$, where $z$ is any word of length $m$ differing from $y$ in all positions; then $C$ is in $\mathcal{U}_{m b \sigma}$. Now it is the case that $(p z y p[m+1 \cdots|p|] y z \leftarrow p y z p y z)$ is in $\tau_{\mathbf{b}}\left(m, \ell_{C}+|s p|+1\right)$ by deleting the word $y$ in $p y z p y z$, which implies that $p y z=p z y$; a contradiction.

In the second case, $s=s_{1} a$, for some word $s_{1}$, and $|p| \geq m$, but $p$ is of the form $p_{1} a p_{2}$ with $\left|p_{1}\right|<m$. Let $C$ be any code in $\mathcal{U}_{m \boldsymbol{b} \sigma}$ with $\ell_{C}=2 m|s p|-|s p|+m+\left|p_{1}\right|$, and let $v$ be any word in $C$. The word $(p v s)^{2|s p|+3}$ is in $(p C s)^{*}$ and can be written as $\left(p v s_{1} a\right)\left(p_{1} a p_{2} v s\right) p_{1} z$, where $z=a p_{2} v s(p v s)^{2|s p|}$. Then, $z$ can be written as $z_{1} \cdots z_{2|s p|} y$ such that $|y|=m$ and each $z_{i}$ is of length $m+\ell_{C}+|s p|$. Now consider the word $\left(p v s_{1} a\right) p_{2} v s p_{1} z^{\prime}$ such that $\left(p v s_{1} a\right) p_{2} v s p_{1}$ obtains from $\left(p v s_{1} a\right)\left(p_{1} a p_{2} v s\right) p_{1}$ by deleting the word $a p_{1}$ and $z^{\prime}$ obtains from $z_{1} \cdots z_{2|s p|} y$ by deleting the suffix $y$ and the prefix of length $m$ of every $z_{i}$. Then, $|z|-\left|z^{\prime}\right|=(2|s p|+1) m$. Moreover, it follows that $\left(\left(p v s_{1} a\right) p_{2} v s p_{1} z^{\prime} \leftarrow\left(p v s_{1} a\right)\left(p_{1} a p_{2} v s\right) p_{1} z\right)$ is in the channel $\tau_{\boldsymbol{b}}\left(m, \ell_{C}+|s p|+1\right)$ and the assumption about $(p, s)$ implies that $\left(p_{2} v s p_{1} z^{\prime} \leftarrow p_{1} a p_{2} v s p_{1} z\right)$ is also in the channel. Let $w=p_{1} a p_{2} v \operatorname{sp}_{1} z$. By Lemma 6

$$
\begin{aligned}
|w| & -\left|p_{2} v s p_{1} z^{\prime}\right| \\
& \leq m\left\lfloor|w| /\left(\ell_{C}+|s p|+m\right)\right\rfloor+\min \left\{m,|w| \%\left(\ell_{C}+|s p|+m\right)\right\}
\end{aligned}
$$

which gives

$$
1+\left|p_{1}\right|+|z|-\left|z^{\prime}\right| \leq m(2|s p|+1)+\left|p_{1}\right| .
$$

This is impossible, however, when we recall that $|z|-\left|z^{\prime}\right|=m(2|\operatorname{sp}|+1)$.

In the third case, $s=s_{1} a$, for some word $s_{1}$, and $|p|<m$. This case can be eliminated by considering the code

$$
C=\left\{a^{\ell} C, a^{\ell} C^{-(m+1)} b^{m+1}\right\}
$$


in $\mathcal{U}_{m \boldsymbol{b} \sigma}$, with $\ell_{C}=2|s| m+m-|s|$ and $b \in X \backslash\{a\}$, and the word $\left(p a^{\ell} C s\right)^{2|s|+3}$ and then continuing as in the second case. We leave the details to the reader.

\section{THE CASE OF SCATTERED ERRORS}

Our first result gives a set of error-detectors for $m \boldsymbol{s} \tau$, for any error type $\tau$. Then, we show that, for $\tau \in\{\iota, \delta,(\sigma \odot \delta),(\sigma \odot \iota)\}$, the result can be improved in terms of the redundancy of the error-detectors.

Proposition 7: Let $m \boldsymbol{s} \tau$ be a scattered-error specification and let $(p, s)$ be a pair of words. If $p \in u^{m} X^{*}$, for some word $u$ with $\operatorname{per}(u)>m$, then $(p, s)$ is a uniform error-detector for $m \boldsymbol{s} \tau$ with delay 1 and offset $m|u|$.

Proof: Assume $p=u^{m} x$ for some word $x$ and consider a code $C$ in $\mathcal{U}_{m \boldsymbol{s} \tau}$. Let

$$
\gamma=\tau_{\mathbf{s}}\left(m, \ell_{C}+|s p|+m|u|\right)
$$

and take any $\left(p v_{1} s u^{m} x v_{2} s y \leftarrow p w_{1} s u^{m} x w_{2} s u\right)$ in $\gamma$, where $v_{1}, v_{2}$, $w_{1}, w_{2} \in C$ and $y \in X^{*}$ and $u \in(p C s)^{*}$. We need to show that $v_{1}=w_{1}$ and $\left(u^{m} x v_{2} s y \leftarrow u^{m} x w_{2} s u\right) \in \gamma$. We agree to write $u_{i}$ for the $i$ th factor $u$ of $u^{m}$, where $i \in\{1, \ldots, m\}$. Suppose that $p w_{1} s$ results in some word $z_{1}$, each $u_{i}$ results in some word $u_{i}^{\prime}$, and $x w_{2} s u$ results in some word $z_{2}$ such that $z_{1} u_{1}^{\prime} \cdots u_{m}^{\prime} z_{2}=p v_{1} s u^{m} x v_{2} s y$. If $\left|z_{1}\right|=\left|p v_{1} s\right|$, then $z_{1}=p v_{1} s$ and we are done. So assume that $d \geq 1$, where $d=\left\|z_{1}|-| p v_{1} s\right\|$; then, at least $d$ errors occur in $p w_{1} s$ and, therefore, $d \leq m$. Moreover, there is $k \in\{1, \ldots, m\}$ such that there is no error in $u_{k}$, namely, $u_{k}^{\prime}=u_{k}$, and there are at most $m-d$ errors in $u_{1} \cdots u_{k-1}$. Then, $z_{1} u_{1}^{\prime} \cdots u_{k-1}^{\prime} u_{k}^{\prime}$ obtains from $p w_{1} s u_{1} \cdots u_{k-1} u_{k}$ and

$$
\begin{aligned}
\left|p w_{1} s u_{1} \cdots u_{k-1} u_{k}\right|-m & \leq\left|z_{1} u_{1}^{\prime} \cdots u_{k-1}^{\prime} u_{k}^{\prime}\right| \\
& \leq\left|p w_{1} s u_{1} \cdots u_{k-1} u_{k}\right|+m .
\end{aligned}
$$

At the same time, as the word $z_{1} u_{1}^{\prime} \cdots u_{k-1}^{\prime} u_{k}^{\prime}$ is a prefix of the word $p v_{1} s u_{1} \cdots u_{k} x v_{2} s y$, it follows that $u_{k}^{\prime}$ is a factor of $p v_{1} s u_{1} \cdots u_{k} x v_{2} s y$ such that, either $u_{k}^{\prime}$ begins in the factor $u_{k}$ of the word $p v_{1} s u_{1} \cdots u_{k} x v_{2} s y$ (when $\left|z_{1} u_{1}^{\prime} \cdots u_{k-1}^{\prime} u_{k}^{\prime}\right| \geq$ $\left.\left|p w_{1} s u_{1} \cdots u_{k-1} u_{k}\right|\right)$, or $u_{k}^{\prime}$ ends in the factor $u_{k}$ of the word $p v_{1} s u_{1} \cdots u_{k} x v_{2} s y$ (when $\left|z_{1} u_{1}^{\prime} \cdots u_{k-1}^{\prime} u_{k}^{\prime}\right| \leq$ $\left.\left|p w_{1} s u_{1} \cdots u_{k-1} u_{k}\right|\right)$. In either case, as $u_{k}^{\prime}=u_{k}=u$ and the period of $u$ is greater than $m$, it follows that the factor $u_{k}^{\prime}$ coincides with the factor $u_{k}$; therefore,

$$
\left|z_{1} u_{1}^{\prime} \cdots u_{k-1}^{\prime} u_{k}^{\prime}\right|=\left|p v_{1} s u_{1} \cdots u_{k-1} u_{k}\right| .
$$

This implies that

$$
\left(u_{k+1} \cdots u_{m} x v_{2} s y \leftarrow u_{k+1} \cdots u_{m} x w_{2} s u\right) \in \gamma
$$

and

$$
\left(p v_{1} s u_{1} \cdots u_{k} \leftarrow p w_{1} s u_{1} \cdots u_{k}\right) \in \gamma
$$

The former relation gives $\left(u^{m} x v_{2} s y \leftarrow u^{m} x w_{2} s u\right) \in \gamma$ and the latter one implies that $p v_{1} s u_{1} \cdots u_{k}$ obtains from $p w_{1} s u_{1} \cdots u_{k}$ using at most $m \boldsymbol{s} \tau$. Moreover, Lemma 2 implies that $v_{1}$ obtains from $w_{1}$ using at most $m \boldsymbol{s} \tau$ and, therefore, $v_{1}=w_{1}$.

The preceding statement allows us to define various error-detectors for any scattered-error specification. The most efficient error-detectors based on this method are those of the form $\left(u^{m}, \lambda\right)$, where $u$ is an unbordered word of length $m+1$.

In [7], it is shown that the coded language $\left(0^{m} X^{\ell-2 m-1} 1\right)^{*}$ is error detecting for $\delta_{\boldsymbol{s}}(m, \ell)$ with delay 0 , and for $\iota_{\boldsymbol{s}}(m, \ell)$ with delay 1 . With the terminology of the present correspondence, it follows that the pair $\left(0^{m}, 1\right)$ is a uniform $m \boldsymbol{s} \delta$-detector with delay 0 and offset $m$, and a uniform $m \boldsymbol{s} \iota$-detector with delay 1 and offset $m$. Moreover, by Proposition $3,\left(0^{m}, 1\right)$ is an error-detector with optimal redundancy. Unlike the case of burst error-detectors, where the offset can be independent of $m$, the offset $m$ of $\left(0^{m}, 1\right)$ cannot be improved.

Proposition 8: If the pair $\left(0^{m}, 1\right)$ is a uniform $m \boldsymbol{s} \delta$-detector with delay 0 and offset $t$, then $t \geq m$.

Proof: For the sake of contradiction assume that the offset $t<m$ and consider the code $C=\left\{w_{1}, w_{2}, w_{3}\right\}$, where $w_{1}=0^{m}(10)^{k}$, $w_{2}=0^{m-1}(10)^{k} 1, w_{3}=1^{2 k+m}, k=1+\lfloor m / 2\rfloor$. Then, $C \in \mathcal{U}^{m}$. Now consider the words $0^{m} w_{1} 10^{m} w_{3} 1$ and $0^{m-1} w_{1} 1 w_{3} 1$. Then,

$$
\left(0^{m-1} w_{1} 1 w_{3} 1 \leftarrow 0^{m} w_{1} 10^{m} w_{3} 1\right) \in \delta_{\boldsymbol{s}}\left(m, \ell_{C}+m+1+t\right) .
$$

Moreover, as $0^{m-1} w_{1} 1 w_{3} 1 \in 0^{m} w_{2} 1 X^{*}$ and $\left(0^{m}, 1\right)$ has delay 0 , it follows that $w_{2}=w_{1}$ which is impossible.

Before we present uniform error-detectors for $(\sigma \odot \iota)$ and $(\sigma \odot \delta)$, we establish certain notation and utility results some of which are of interest in their own right.

Every nonempty word $w$ can be written in the form $a_{1}^{n_{1}} a_{2}^{n_{2}} \cdots a_{r}^{n_{r}}$, where $r$ and $n_{1}, \ldots, n_{r}$ are positive integers, $a_{1}, \ldots, a_{r} \in X$, and $a_{i} \neq a_{i+1}$ for all $i \in\{1, \ldots, r-1\}$. In this case, each factor $a_{i}^{n_{i}}$ of $w$ is called a run. We use the symbol $w\langle i\rangle$ to denote the $i$ th run of $w$. Now let $r, n$ be positive integers with $r \geq 2$. An $(r, n)$-alternating word is a word $w$ of the form $a_{1}^{n} a_{2}^{n} \cdots a_{r}^{n}$, where $w\langle i\rangle=a_{i}^{n}$ for all $i \in\{1, \ldots, r\}$. In the sequel, when we use the term $(r, n)$-alternating word we assume without mention that $r$ and $n$ are positive integers with $r \geq 2$.

Lemma 7: Assume that $X=\{0,1\}$ and $a_{1}^{n} \cdots a_{r}^{n}$ is an $(r, n)$-alternating word. Consider the word $w=a_{1}^{n-t} a_{2}^{n} \cdots a_{r}^{n}$, where $t$ is a positive integer with $t<n$, and suppose that a prefix $p$ of $a_{1}^{n} \cdots a_{r}^{n}$ obtains from $w$ using $k_{1} \boldsymbol{s} \sigma$ and $k_{2} \boldsymbol{s} \delta$, for some nonnegative integers $k_{1}$ and $k_{2}$. Then, in obtaining $p$ from $w$, the following statements hold true about $k_{1} \boldsymbol{s} \sigma$ and $k_{2} \boldsymbol{s} \delta$.

1) If fewer than $|w\langle i\rangle|$ errors are used in $w\langle i\rangle$ for every $i=1, \ldots, r$, then $k_{1} \geq r-1$.

2) If there is an $i \in\{1, \ldots, r-1\}$ such that $|w\langle i\rangle|$ errors are used in $w\langle i\rangle$, then $k_{1}+k_{2} \geq 2 n-t$.

Proposition 9: Assume $X=\{0,1\}$ and let $k$ be a nonnegative integer. Let $u$ be an $(r, n)$-alternating word and let $v$ be a proper and nonempty suffix of $u$.

1) If a prefix of $u$ obtains from $v$ using $k s(\sigma \odot \delta)$, then $k \geq \min \{r-$ $1,2 n-(|u|-|v|)\}$.

2) If $v$ obtains from a prefix of $u$ using $k \boldsymbol{s}(\sigma \odot \iota)$, then $k \geq \min \{r-$ $1,2 n-(|u|-|v|)\}$.

Proposition 10: Assume $X=\{0,1\}$ and let $k$ be a nonnegative integer. Let $v$ be a nonempty word and let $u$ be an $(r, n)$-alternating word.

1) If a prefix of $v u$ obtains from $u$ using $k s(\sigma \odot \delta)$, then $k \geq$ $\min \{r-1,2 n-|v|\}$.

2) If $u$ obtains from a prefix of $v u$ using $k \boldsymbol{s}(\sigma \odot \iota)$, then $k \geq$ $\min \{r-1,2 n-|v|\}$.

Proposition 11: Let $m$ be a positive integer, let $n=\lfloor m / 2\rfloor+1$, and assume $X=\{0,1\}$. For any $(r, n)$-alternating word $a_{1}^{n} \cdots a_{r}^{n}$ the following statements hold true.

1) If $r \geq m+1$, then $\left(\lambda, a_{1}^{n} \cdots a_{r}^{n}\right)$ is a uniform error-detector for $m \boldsymbol{s}(\sigma \odot \delta)$ with delay 1 and offset $2 n^{2}$.

2) If $r \geq 2 n+1$, then $\left(\lambda, a_{1}^{n} \cdots a_{r}^{n}\right)$ is a uniform error-detector for $m \boldsymbol{s}(\sigma \odot \delta)$ with delay 1 and offset 0 . 
Proof: We prove both statements simultaneously. Moreover, we only consider the case where $m \geq 2$ and, therefore, $n \geq 2$. One can verify that the claim also holds for $m=1$. Let $C$ be any code in $\mathcal{U}_{m s \sigma}$, let $s=a_{1}^{n} \cdots a_{r}^{n}$, and let $\gamma=(\sigma \odot \delta)_{s}\left(m, \ell_{C}+|s|+t\right)$, where $t=2 n^{2}$ if $r=2 n$, and $t=0$ if $r \geq 2 n+1$. Suppose $\left(v_{1} s v_{2} s y \leftarrow w_{1} s w_{2} s u\right) \in \gamma$ for some words $v_{1}, v_{2}, w_{1}, w_{2} \in C$ and $y \in X^{*}$ and $u \in(C s)^{*}$. We need to show that $v_{1}=w_{1}$ and $\left(v_{2} s y \leftarrow w_{2} s u\right) \in \gamma$. If there are no errors in $w_{1} s$, then we are done. If there are only substitution errors in $w_{1} s$, then they all occur in $w_{1}$ and, therefore, $v_{1}$ obtains from $w_{1}$ using up to $m$ substitutions which contradicts the fact that $C \in \mathcal{U}_{m s \sigma}$. Now assume there is at least one deletion in $w_{1} s$. In particular let $d_{1}$ be the number of deletions in $w_{1}$, let $d_{2}$ be the number of deletions in the first $s$ of $w_{1} s w_{2} s$, and let $d_{3}$ be the number of deletions in $w_{2}$. Then $d_{1}+d_{2} \geq 1$.

If $d_{1} \geq 1$, then $w_{1}$ results in a prefix of length $\ell_{C}-d_{1}$ of $v_{1}$ and $s$ results in a prefix of $x s$, where $x$ is the suffix of length $d_{1}$ of $v_{1}$. Then there are at least $\min \{r-1,2 n-|x|\} \geq \min \left\{m, 2 n-d_{1}\right\}$ errors in $s$ and, therefore, at least $d_{1}+\min \left\{m, 2 n-d_{1}\right\}$ errors in $w_{1} s$ which is of length $\ell_{C}+|s|$. This is a contradiction, however, as $2 n>m$. So in the rest of the proof we assume that $d_{1}=0$ and $d_{2} \geq 1$; then, $d_{2}+d_{3} \leq m$. Also, $w_{1} s w_{2}$ results in a prefix of length $\ell_{C}+|s|+\ell_{C}-\left(d_{2}+d_{3}\right)$ of $v_{1} s v_{2}$ and the second $s$ of $w_{1} s w_{2} s$ results in a prefix of $x s$, where $x$ is the suffix of length $d_{2}+d_{3}$ of $v_{1} s v_{2}$. Then, there are at least $2 n-|x|=2 n-\left(d_{2}+d_{3}\right)$ errors in the second $s$ which gives at least $m+1$ errors in $s w_{2} s$ whose length is $\ell_{C}+|s|+|s|$. Then a contradiction arises when $r=2 n$ and $t=2 n^{2}$, and the first statement is proved. So in the sequel we assume that $r \geq 2 n+1$ and $t=0$; that is, the channel $\gamma$ is $(\sigma \odot \delta)_{s}\left(m, \ell_{C}+|s|\right)$. Consider the run $s\langle i\rangle=a_{i}^{n}$ of $s$ containing the first of the $d_{2}$ deletions.

Let $w$ be the word $a_{i}^{n} a_{i+1}^{n} \cdots a_{r}^{n} w_{2} a_{1}^{n} \cdots a_{i-1}^{n}$; then $|w|=\ell_{C}+|s|$ and $w$ results in a prefix of the word $a_{i}^{n} a_{i+1}^{n} \cdots a_{r}^{n} v_{2} a_{1}^{n} \cdots a_{i-1}^{n}$ using $k \boldsymbol{s}(\sigma \odot \delta)$, for some integer $k$ with $d_{2}+d_{3} \leq k<2 n$. We shall show that our assumptions lead to a contradiction. First note that the word $z=a_{i}^{n-1} a_{i+1}^{n} \cdots a_{r}^{n} w_{2} a_{1}^{n} \cdots a_{i-1}^{n}$ obtains from $w$ using the first deletion in $a_{i}^{n}$ and then a prefix of $a_{i}^{n} \cdots a_{r}^{n} v_{2} a_{1}^{n} \cdots a_{i-1}^{n}$ obtains from $z$ using $(k-1) \boldsymbol{s}(\sigma \odot \delta)$. Hence, if $i \leq r-1$ then there are at least $\min \{r-i, 2 n-1\}$ errors in the prefix $a_{i}^{n-1} a_{i+1}^{n} \cdots a_{r}^{n}$ of $z$. Also, note that $a_{1}^{n} \cdots a_{i-1}^{n}$ results in a prefix of $v a_{1}^{n} \cdots a_{i-1}^{n}$, where $v$ is the suffix of length $d_{2}+d_{3}$ of $v_{2}$; that is, $|v|=d_{2}+d_{3}$. Hence, if $i \geq 3$, there are at least $\min \left\{i-2,2 n-\left(d_{2}+d_{3}\right)\right\}$ errors in the suffix $a_{1}^{n} \cdots a_{i-1}^{n}$ of $z$.

If $i \leq 2$, then $k-1 \geq \min \{r-2,2 n-1\}$ which implies that $k \geq 2 n$; a contradiction. If $i \geq 3$ and $i-2 \geq 2 n-\left(d_{2}+d_{3}\right)$, then $k \geq\left(d_{2}+d_{3}\right)+2 n-\left(d_{2}+d_{3}\right)$ which is a contradiction. Finally, if $i \geq 3$ and $i-2<2 n-\left(d_{2}+d_{3}\right)$, then $k-1 \geq \min \{r-i, 2 n+1\}+(i-2)$ which implies that $k \geq 2 n$; a contradiction.

Using similar arguments as above, one can verify that also the following statement holds true.

Proposition 12: Let $m$ be a positive integer, let $n=\lfloor m / 2\rfloor+1$, and assume $X=\{0,1\}$. For any $(r, n)$-alternating word $a_{1}^{n} \cdots a_{r}^{n}$, we have the following statements.

1) If $r \geq m+1$, then $\left(\lambda, a_{1}^{n} \cdots a_{r}^{n}\right)$ is a uniform error-detector for $m \boldsymbol{s}(\sigma \odot \iota)$ with delay 1 and offset $2 n^{2}$.

2) If $r \geq 2 n+1$, then $\left(\lambda, a_{1}^{n} \cdots a_{r}^{n}\right)$ is a uniform error-detector for $m \boldsymbol{s}(\sigma \odot \iota)$ with delay 1 and offset 0 .

\section{DISCUSSION}

We have presented an analysis of the method of separators for detecting synchronization (and substitution) errors in the messages of a coded language. For the case of burst errors, we were able to obtain a simple necessary and sufficient condition on the structure of the separators. It would be interesting to find such a condition on separators that detect scattered errors as well. This would allow us to evaluate the various separators for scattered errors designed in the correspondence. We note that this question is related to the problem of frame synchronization in the presence of scattered substitution errors—see [15] for details.

Regarding separators for error correction [1], it is straightforward to define the parameters of redundancy and offset as in this correspondence. On the other hand, the parameter of delay should be defined with some care as the definition of "error correction with finite delay" given in [1] involves automata with output (finite-state machines). If we ignore that parameter for now, the results of [1] on "error-correctors" (separators for error correction) can be rephrased as follows.

1) The pair $\left(0^{m}, 1^{m}\right)$ is a uniform error-corrector for $m \boldsymbol{s} \delta$ with redundancy $2 m$ and offset 1 .

2) The pair $\left(\lambda, 1^{m} 0^{m}\right)$ is a uniform error-corrector for $m \boldsymbol{s} \iota$ with redundancy $2 \mathrm{~m}$ and offset $2 \mathrm{~m}$.

3) The pair $\left(\lambda,\left(1^{m+1} 0^{m+1}\right)^{m+1} 1^{m}\right)$ is a uniform error-corrector for $m \boldsymbol{s}(\sigma \odot \iota \odot \delta)$ and for $m \boldsymbol{s}(\iota \odot \delta)$ with redundancy $2(m+1)^{2}+m$ and offset $2(m+1)^{2}+m$.

If we ignore lower order terms, it follows that, for the same error specification, Levenshtein's error-correctors are twice as long as the errordetectors designed here. Intuitively, this observation is consistent with the view that the amount of redundancy for error correction is (roughly) twice the amount for error detection.

\section{tions. tions.}

Proof of Lemma 1: The "only if" part is obvious. For the "if" part, first assume that $\tau \in\{(\sigma \odot \iota),(\sigma \odot \delta)\}$. Then the statement follows easily when we note that, if $v^{\prime}$ obtains from $v$ using at most $m \boldsymbol{x} \tau$, only substitution errors can occur in $v$. Now assume that $\tau \in\{(\iota \odot \delta),(\sigma \odot$ $\iota \odot \delta)\}$; then $\boldsymbol{x}=\boldsymbol{b}$ and $v=p u s$ and $v^{\prime}=p u^{\prime} s$ such that $\left(u^{\prime} \leftarrow u\right)$ is in $B_{m}(\tau)$. As $|v|=\left|v^{\prime}\right|$, one has that $|u|=\left|u^{\prime}\right|$ which implies that $D_{\sigma}\left(v, v^{\prime}\right) \leq m$ and, therefore, $\left(u^{\prime} \leftarrow u\right)$ is in $B_{m}(\sigma)$. Hence, $v^{\prime}$ obtains from $v$ using at most $m \boldsymbol{x} \sigma$.

Proof of Lemma 2: If $v=v^{\prime}$ then the statement is obvious. So assume $v \neq v^{\prime}$; then $\tau \notin\{\iota, \delta\}$ and, either $\tau=\sigma$ or $\tau$ contains at least two different error types. If $m \boldsymbol{x} \tau$ is other than $m \boldsymbol{s}(\iota \odot \delta)$ and $m \boldsymbol{s}(\sigma \odot \iota \odot \delta)$, then the statement follows easily from Lemma 1. If $m \boldsymbol{x} \tau$ is either of $m \boldsymbol{s}(\iota \odot \delta)$ and $m \boldsymbol{s}(\sigma \odot \iota \odot \delta)$, then the statement follows from the fact that $D_{\tau}\left(v, v^{\prime}\right)=D_{\tau}\left(p v s, p v^{\prime} s\right)$ [1].

Proof of Lemma 3: For the first claim, we write $u$ in the form $x y$ for some words $x$ and $y$ with $|x|=\left|u^{\prime}\right|$. Then the claim follows when we note that $u^{\prime}$ obtains from $u$ by deleting $y$ and substituting $D_{\sigma}\left(u^{\prime}, x\right)$ symbols in $x$. For the second claim, if no deletions are used to obtain $u^{\prime}$ from $u$ then we are done. If at least one deletion is used then there must be at least two insertions in $u$-so that $\left|u^{\prime}\right|>|u|$ —and, therefore, $\left|u^{\prime}\right|-|u| \leq m-2$. In this case, $u^{\prime}$ can be written as $x y$ with $|x|=|u|$ and $|y| \leq m-2$. Then the claim follows when we note that $u^{\prime}$ obtains from $u$ by substituting $D_{\sigma}(u, x)$ symbols in $u$ and then inserting $y$ at the end.

For the last claim, let $X=\{0,1\}$ and let $k \leq m$ be such that $u^{\prime}$ obtains from $u$ using $k s(\sigma \odot \iota \odot \delta)$. Without loss of generality, suppose $u[1]=0$. First assume that, for all $i=1, \ldots,|u|, u^{\prime}[i] \neq u[i]$ and $u^{\prime}[i+1] \neq u[i]$. Then it follows that $u=0^{|u|}$ and $u^{\prime}=1^{|u|+1}$. 
In this case, to obtain $u^{\prime}$ from $u$, all symbols of $u$ must be deleted or substituted and at least a 1 must be inserted. Hence, $k \geq|u|+1$ which implies $|u|<m$. Then, $u^{\prime}$ obtains from $u$ by substituting $u$ with $1^{|u|}$ and inserting a 1 at the end. Therefore, $u^{\prime}$ obtains from $u$ using at most $m \boldsymbol{s}(\sigma \odot \iota)$. Now assume that there is $i \in\{1, \ldots,|u|\}$ such that $u^{\prime}[i]=u[i]$ or $u^{\prime}[i+1]=u[i]$. In the former case, suppose $i$ is the smallest with this property. Then, $u^{\prime}$ can be written as $u_{1}^{\prime} u[i] u_{2}^{\prime}$ and $u$ as $u_{1} u[i] u_{2}$ such that $\left|u_{1}^{\prime}\right|=\left|u_{1}\right|$ and $D_{\sigma}\left(u_{1}^{\prime}, u_{1}\right)=\left|u_{1}\right|$. In the latter case, suppose $i$ is the largest with the property $u^{\prime}[i+1]=u[i]$. Then, $u^{\prime}$ can be written as $u_{1}^{\prime} u[i] u_{2}^{\prime}$ and $u$ as $u_{1} u[i] u_{2}$ such that $\left|u_{2}^{\prime}\right|=\left|u_{2}\right|$ and $D_{\sigma}\left(u_{2}^{\prime}, u_{2}\right)=\left|u_{2}\right|$. In either case, $u^{\prime}$ obtains from $u$ using at most $\left(\left|u_{1}\right|+\left|u_{2}\right|\right) \boldsymbol{s} \sigma$ and then $1 \boldsymbol{s} \iota$, which proves the claim.

\section{Proof of Proposition 2: Assume that}

$$
B_{m}(\sigma \odot \iota \odot \delta)=B_{m}(\sigma \odot \delta) \cup B_{m}(\sigma \odot \iota) .
$$

First, we show that $m \leq 4$. Indeed, suppose $m \geq 5$. Then there are positive integers $q$ and $r$ such that $m=3 q+r$ and $r \geq 2 q$ (for example, $q=1$ and $r=m-3$ ). Consider the words $u=0^{q+r} 1^{q} 0^{q}$ and $u^{\prime}=1^{2 q+r} 0^{q+r} 1^{q}$. Then, $|u|=m,\left|u^{\prime}\right|=|u|+q+r$, and $u^{\prime}$ obtains from $u$ using $(2 q+r) \boldsymbol{s} \iota$ and $q \boldsymbol{s} \delta$; therefore, $\left(u^{\prime} \leftarrow u\right) \in$ $B_{m}(\sigma \odot \iota \odot \delta)$. Suppose now that $u^{\prime}$ obtains from $u$ using $k \boldsymbol{s}(\sigma \odot \iota)$, for some integer $k$ with $q+r \leq k \leq m$. Then also $u$ obtains from $u^{\prime}$ using $k \boldsymbol{s}(\sigma \odot \delta)$ which implies that there is a word $z$ such that $z$ obtains from $u^{\prime}$ using $(q+r) \boldsymbol{s} \delta$ and $u$ obtains from $z$ using $(k-q-$ $r) \boldsymbol{s} \sigma$ - see [6] for instance. Then, $z$ is of the form $1^{2 q+r-i} 0^{q+r-j} 1^{q-t}$ for some nonnegative integers $i, j, t$ with $t \leq q$ and $i+j+t=$ $q+r$. By distinguishing two cases depending on whether $\left|1^{q} 0^{q}\right| \leq$ $\left|0^{q+r-j} 1^{q-t}\right|$, it follows that $D_{\sigma}(z, u)>2 q$; therefore, $k>q+r+$ $2 q=m$ which is a contradiction. Hence, $m \leq 4$. To complete the "only if" part we need to show that, if $m \in\{3,4\}$, then $X$ must be $\{0,1\}$. For the sake of contradiction, suppose there is a symbol $a \in$ $X \backslash\{0,1\}$ and consider the words $u=01 a^{m-2}$ and $u^{\prime}=1 a 0^{m-1}$, with $m \in\{3,4\}$. Then, $u^{\prime}$ obtains from $u$ using $m \boldsymbol{s}(\sigma \odot \iota \odot \delta)$ but $u$ cannot obtain from $u^{\prime}$ using at most $m \boldsymbol{s}(\sigma \odot \delta)$; therefore, $u^{\prime}$ cannot obtain from $u$ using at most $m \boldsymbol{s}(\sigma \odot \iota)$.

For the converse, we note first that, obviously

$$
B_{m}(\sigma \odot \delta) \cup B_{m}(\sigma \odot \iota) \subseteq B_{m}(\sigma \odot \iota \odot \delta) .
$$

For the reverse inclusion, let $\left(u^{\prime} \leftarrow u\right) \in B_{m}(\sigma \odot \iota \odot \delta)$ and consider the following cases.

Case 1: $m=1$. One verifies by inspection that $\left(u^{\prime} \leftarrow u\right) \in$ $B_{1}(\sigma \odot \delta) \cup B_{1}(\sigma \odot \iota)$.

Case 2: $m=2$. In this case, $2 m-2=2$ and it follows from Proposition 1 that $\left(u^{\prime} \leftarrow u\right) \in B_{2}(\sigma \odot \delta) \cup B_{2}(\sigma \odot \iota)$.

Case 3: $m=3$ and $X=\{0,1\}$. We use Lemma 3. If $\left|u^{\prime}\right| \leq|u|$ then $\left(u^{\prime} \leftarrow u\right) \in B_{m}(\sigma \odot \delta)$. If $\left|u^{\prime}\right|>|u|$ there are two subcases. If no deletions are used to obtain $u^{\prime}$ from $u$ then clearly $\left(u^{\prime} \leftarrow u\right) \in$ $B_{m}(\sigma \odot \iota)$. If a deletion is used in obtaining $u^{\prime}$ from $u$ then $\left|u^{\prime}\right|=$ $|u|+1$ and, therefore, $\left(u^{\prime} \leftarrow u\right) \in B_{m}(\sigma \odot \iota)$.

Case 4: $m=4$ and $X=\{0,1\}$. We use again Lemma 3. If $\left|u^{\prime}\right| \leq$ $|u|$ then $\left(u^{\prime} \leftarrow u\right) \in B_{m}(\sigma \odot \delta)$. So assume $\left|u^{\prime}\right|>|u|$. If $\left|u^{\prime}\right|=|u|+1$ then we are done as in Case 3. If $\left|u^{\prime}\right|>|u|+1$ then, as $|u| \leq m=4$, it follows that $\left|u^{\prime}\right|=|u|+2$. If no deletion is used in $u$ then we are done. If a deletion is used in $u$ then it is the only one and there are also exactly three insertions to obtain $u^{\prime}$. Hence, $u^{\prime}$ obtains from $u$ using $3 \boldsymbol{s} \iota$ and $1 \boldsymbol{s} \delta$. Then, using a long case distinction, one can verify that again $\left(u^{\prime} \leftarrow u\right) \in B_{m}(\sigma \odot \iota)$.

Proof of Proposition 5: The assumptions about $m \boldsymbol{b} \tau$ imply that $\tau$ is in $\{(\sigma \odot \delta),(\sigma \odot \iota),(\iota \odot \delta),(\sigma \odot \iota \odot \delta)\}$. Assume that $(p, s)$ is an $m \boldsymbol{b} \tau$-detector with redundancy $m+1$, offset 0 , and delay $d$, for some nonnegative integer $d$. We shall obtain a contradiction by constructing a code $C \in \mathcal{U}_{m \boldsymbol{b} \tau}$ such that $(p C s)^{*}$ is not error detecting for $\tau_{\boldsymbol{b}}\left(m, \ell_{C}+|p s|\right)$ with delay $d$. First, write the word $s p$ in the form $a x b$, for some $a, b \in X$ and $x \in X^{*}$, and let $C=\{x b y, y a x\}$, where $y=1^{m} 0^{m} 1^{m}$. Then, $C$ is error detecting for $\tau_{\boldsymbol{b}}\left(m, \ell_{C}\right)$ and, therefore, $C \in \mathcal{U}_{m \boldsymbol{b} \tau}$. Now let $w$ be the word $(p x b y s)^{d+3}$ if $\tau$ contains $\delta$,

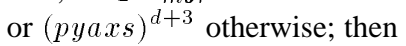

$$
w=p x b y(a x b) x b y(a x b)(x b y(a x b))^{d} x b y s
$$

or

$$
w=\operatorname{pyax}(a x b) \operatorname{yax}(a x b)(\operatorname{yax}(a x b))^{d} \text { yaxs. }
$$

Consider also the word $z=$

$$
\begin{cases}\operatorname{pxby}(a) x b y(a x a)(x b y(a x a))^{d} x b y s, & \text { if } \tau \text { contains } \delta \\ \operatorname{pyax}(a x b x b) y a x(b x b)(y a x(b x b))^{d} y a x s, & \text { otherwise. }\end{cases}
$$

Then, $z$ is equal to either

$$
(\text { pxbys })(\text { pyaxs })(\text { pyaxs })^{d} \text { pys }
$$

or

$$
(\text { pyaxs })(\text { pxbys })(\text { pxbys })^{d} \text { pxbyaxs. }
$$

If $\tau$ contains $\delta$, then $z$ obtains from $w$ by deleting the suffix $x b$ in the prefix pxbyaxb of $w$, and then by transforming $d+1 b$ 's in $w$ to $a$ 's using the errors permitted. If $\tau$ does not contain $\delta$, then $z$ obtains from $w$ by inserting the word $x b$ at the end of the prefix pyaxa of $w$, and then by transforming $d+1 a$ 's in $w$ to $b$ 's using the errors permitted. In either case, it follows that $(z \leftarrow w) \in \tau_{\boldsymbol{b}}\left(m, \ell_{C}+m+1\right)$ which implies that pyaxs $=$ pxbys; a contradiction.

Proof of Lemma 6: Let $q=\lfloor|w| /(\ell-1+m)\rfloor$. Then $w$ can be written as $w_{1} \cdots w_{q} x$ with $|x|=|w| \%(\ell-1+m)$ and $\left|w_{i}\right|=\ell-$ $1+m$. Also $z$ can be written as $w_{1}^{\prime} \cdots w_{q}^{\prime} x^{\prime}$ such that $\left(x^{\prime} \leftarrow x\right)$ and every $\left(w_{i}^{\prime} \leftarrow w_{i}\right)$ are in the channel. As $\left|w_{i}\right|=\ell-1+m$ and $\ell>m$, in each $w_{i}$ either there are at most two bursts of errors, or three bursts of errors that involve only insertions-the latter case is possible only when $\ell=m+1$. It follows then that the number of deleted symbols in the burst(s) is at most $m$ and, therefore, $\left|w_{i}\right|-\left|w_{i}^{\prime}\right| \leq m$. Now for the word $x$ we have two cases. If $|x| \leq m$ then at most $|x|$ symbols can be deleted in $x$ and, therefore, $|x|-\left|x^{\prime}\right| \leq|x|$. If $|x|>m$ then, as $|x|<\ell-1+m$, one can use the same argument as above to infer that $|x|-\left|x^{\prime}\right| \leq m$. In either case, $|x|-\left|x^{\prime}\right| \leq \min \{|x|, m\}$. Hence, $|w|-|z| \leq q m+\min \{|x|, m\}$ as required.

Proof of Lemma 7: For the first statement, note first that there is a word $y$ such that $y$ obtains from $w$ using $k_{2} \boldsymbol{s} \delta$ and $p$ obtains from $y$ using $k_{1} \boldsymbol{s} \sigma$. Then, $y$ is of the form $a_{1}^{n-s_{1}} a_{2}^{n-s_{2}} \cdots a_{r}^{n-s_{r}}$, where each $s_{i}$ is a nonnegative integer with $s_{i}<n$ and $n-s_{1} \leq n-t$. Then, to get $p$ from $y$ using $k_{1} \boldsymbol{s} \sigma$, consider the fact that at least one symbol from each run of $y$ will appear in $p$ with no error. It follows then that at least the first symbol of each run $y\langle i\rangle$, with $i \in\{2, \ldots, r\}$, will be substituted. Hence, $k_{1} \geq r-1$.

For the second statement, there is a word $y$ such that $y$ obtains from $w$ using $|w\langle i\rangle|$ errors in $w\langle i\rangle$ and $p$ obtains from $y$ using $\left(k_{1}+k_{2}-\right.$ $|w\langle i\rangle|) s(\sigma \odot \delta)$. If $i=1$, then $w\langle i\rangle=a_{1}^{n-t}$ results in $a_{2}^{s}$, for some $s \in\{0, \ldots, n-t\}$; therefore, $y$ begins with a prefix of the form $a_{2}^{s} a_{2}^{n}$. As $a_{1} \neq a_{2}$, obtaining $p$ from $y$ requires at least $n$ errors in $a_{2}^{s} a_{2}^{n}$. Hence, $k_{1}+k_{2} \geq(n-t)+n=2 n-t$. If $i \geq 2$ then $w\langle i\rangle=a_{i}^{n}$ and $y$ will contain a factor of the form $a_{i-1}^{n-t} a_{i-1}^{s} a_{i-1}^{n}$, where $a_{i-1}^{s}$ results from $w\langle i\rangle$ and $s \in\{0, \ldots, n\}$. Thus, to get $p$ from $y$ at least $n-t+s$ errors are needed in the factor $a_{i-1}^{2 n-t+s}$ of $y$. Hence, $k_{1}+k_{2} \geq 2 n-t$.

Proof of Proposition 9: Assume $u=p v$ with $p, v \neq \lambda$. For the first part, suppose that $v$ results in a prefix of $u$ using $k \boldsymbol{s}(\sigma \odot \delta)$. If $2 n \leq|u|-|v|$ we are done. So assume $|u|-|v|<2 n$. If $|u|-|v|=$ $n+t$, for some integer $t$ with $0 \leq t<n$, then $v=a_{2}^{n-t} a_{3}^{n} \cdots a_{r}^{n}$ 
results in a prefix of $a_{1}^{n} \cdots a_{r}^{n}$ using $k \boldsymbol{s}(\sigma \odot \delta)$. But, as $a_{1} \neq a_{2}$, there must be $n-t$ errors in the prefix $a_{2}^{n-t}$ of $v$. Hence, $k \geq n-t=$ $2 n-(n+t)$ as required. Now assume $|u|-|v|=t$ with $t<n$; then $v=$ $a_{1}^{n-t} a_{2}^{n} \cdots a_{r}^{n}$ results in a prefix of $a_{1}^{n} \cdots a_{r}^{n}$ using $k_{1} \boldsymbol{s} \sigma$ and $k_{2} \boldsymbol{s} \delta$, where $k_{1}+k_{2}=k$. We consider three cases. In the first case, no run $v\langle i\rangle$ of $v$ has $|v\langle i\rangle|$ errors. Then, $k_{1} \geq r-1$ which implies $k \geq r-1$ as required. In the second case, some run $v\langle i\rangle$, with $i \in\{1, \ldots, r-1\}$, has $|v\langle i\rangle|$ errors. Then, $k \geq 2 n-t$ as required. In the third case, there are $|v\langle n\rangle|$ errors in the last run $a_{r}^{n}$ of $v$ and, therefore, $k \geq n$. If $r=2$ we are done. If $r \geq 3$ then note that the word $a_{1}^{n-t} a_{2}^{n} \cdots a_{r-1}^{n}$ results in a prefix of $a_{1}^{n} a_{2}^{n} \cdots a_{r-1}^{n}$ using $(k-n) \boldsymbol{s}(\sigma \odot \delta)$ and, by Lemma 7, it follows that $k-n \geq \min \{r-2, n-t\}$. Hence, $k \geq \min \{r-1,2 n-t\}$ as required.

For the second part, assume that $v$ results from a prefix of $u$ using $k \boldsymbol{s}(\sigma \odot \iota)$. Then the prefix of $u$ results from $v$ using $k \boldsymbol{s}(\sigma \odot \delta)$ and the claim follows from the first part.

Proof of Proposition 10: We only show the first part; the second part follows easily as in the previous proposition. If $u$ results in a prefix of $v$ then at least $|u|-|v|$ deletions must be used in $u$; therefore, $k \geq$ $2 n-|v|$ as required. So assume that $u$ results in $v p$, where $p$ is a nonempty prefix of $u$. Then, there is a prefix $x$ of $u$, with $|x| \geq|v|$, such that $v$ obtains from $x$ using $k_{1} \boldsymbol{s}(\sigma \odot \delta)$, and $p$ obtains from $y$ using $k_{2} \boldsymbol{s}(\sigma \odot \delta)$, where $k_{1}+k_{2}=k$ and $y$ is such that $u=x y$. As there must be exactly $|x|-|v|$ deletions in $|x|$, we have that $k_{1} \geq|x|-|v|$. If $|x| \geq 2 n$ then we are done. So assume $|x|<2 n$. If $|x|=n+t$ with $0 \leq t<n$ then $x=a_{1}^{n} a_{2}^{t}$ and $y=a_{2}^{n-t} a_{3}^{n} \cdots a_{r}^{n}$ which results in a prefix of $a_{1}^{n} \cdots a_{r}^{n}$. Then, as $a_{1} \neq a_{2}$, there must be $n-t$ errors in the prefix $a_{2}^{n-t}$ of $y$ and, therefore, $k_{2} \geq n-t$. Hence, $k_{1}+k_{2} \geq 2 n-|v|$ as required. Now assume $|x|<n$. Then, $x=a_{1}^{|x|}$, $y=a_{1}^{n-|x|} a_{2}^{n} \cdots a_{r}^{n}$, and a prefix of $a_{1}^{n} \cdots a_{r}^{n}$ obtains from $y$ using $k_{2} \boldsymbol{s}(\sigma \odot \delta)$. This implies that $k_{2} \geq \min \{r-1,2 n-|x|\}$, which, in turn, implies that $k_{1}+k_{2} \geq \min \{r-1,2 n-|v|\}$ as required.

\section{ACKNOWLEDGMENT}

The authors wish to thank the anonymous referees for suggesting references [12] and [15].

\section{REFERENCES}

[1] V. I. Levenshtein, "Binary codes capable of correcting deletions, insertions, and reversals," Sov. Phys.-Dokl.: Cybern. Contr. Theory, vol. 10, pp. 707-710, 1966.

[2] F. F. Sellers, Jr., "Bit loss and gain correction code," IEEE Trans. Inform. Theory, vol. IT-8, pp. 35-38, Jan. 1962.

[3] H. C. Ferreira, W. A. Clarke, A. S. J. Helberg, K. A. S. Abdel-Ghaffar, and A. J. H. Vinck, "Insertion/deletion correction with spectral nulls," IEEE Trans. Inform. Theory, vol. 43, pp. 722-732, Mar. 1997.

[4] M. Lothaire, Algebraic Combinatorics on Words. Cambridge, U.K.: Cambridge Univ. Press, 2002.

[5] L. J. Guibas and A. Odzylko, "Periods in strings," J. Combin. Theory, Ser. A, vol. 30, pp. 19-42, 1981.

[6] S. Konstantinidis, "Relationships between different error-correcting capabilities of a code," IEEE Trans. Inform. Theory, vol. 47, pp. 2065-2069, July 2001.

[7] S. Konstantinidis and A. O'Hearn, "Error-detecting properties of languages," Theor. Comput. Sci., vol. 276, pp. 355-375, 2002.

[8] J. Berstel and D. Perrin, Theory of Codes. Orlando, FL: Academic, 1985
[9] W. W. Peterson and E. J. Weldon, Error Correcting Codes, 2nd ed. Cambridge, MA: MIT Press, 1972.

[10] V. I. Levenshtein, "On perfect codes in the deletion/insertion metric," Discr. Math. and Appl., vol. 2, pp. 241-258, 1992.

[11] G. Tenengolts, "Nonbinary codes correcting single deletion or insertion," IEEE Trans. Inform. Theory, vol. IT-30, pp. 766-769, Sept. 1984.

[12] A. S. J. Helberg and H. C. Ferreira, "On multiple insertion/deletion correcting codes," IEEE Trans. Inform. Theory, vol. 48, pp. 305-308, Jan. 2002.

[13] L. J. Guibas and A. Odzylko, "String overlaps, pattern matching, and nontransitive games," J. Combin. Theory, Ser. A, vol. 30, pp. 183-208, 1981.

[14] H. Morita, A. J. van Wijngaarden, and A. J. H. Vinck, "On the construction of maximal prefix-synchronized codes," IEEE Trans. Inform. Theory, vol. 42, pp. 2158-2166, Nov. 1996.

[15] P. Bylanski and D. G. W. Ingram, Digital Transmission Systems. Herts, U.K.: Peter Peregrinus Ltd., 1976.

Stavros Konstantinidis (M'02) received the B.Sc. degree in mathematics from the University of Athens, Athens, Greece, in 1988 and the M.Sc. and Ph.D. degrees in computer science from the University of Western Ontario, London, ON, Canada, in 1992 and 1996, respectively.

From 1996 to 1998, he was an Assistant Professor of Computer Science at the University of Lethbridge, Lethbridge, AB, Canada. In 1998, he joined the Department of Mathematics and Computing Science at Saint Mary's University, Halifax, NS, Canada, where he currently is an Associate Professor. His research interests include automata, formal languages, and coding theory.

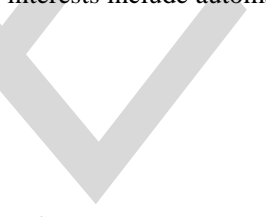

Steven Perron is an undergraduate student at Saint Mary's University, Halifax, NS, Canada. He expects to receive the B.Sc. degree in May 2003. He plans to go to graduate school and study computer science.

His research interests include complexity theory, coding theory, and algorithms.

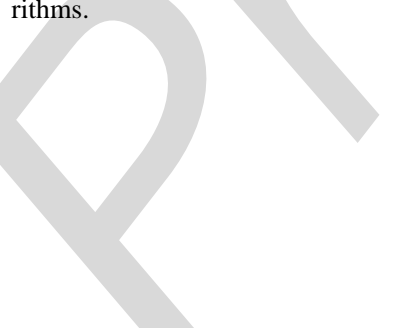

L. Amber Wilcox-O'Hearn received the B.A. degree (with honors) in Russian language from the University of Waterloo, Waterloo, ON, Canada in 1997, and the B.Sc. degree in computing science from Saint Mary's University, Halifax, NS, Canada, in 2002, and is currently a graduate student in the Department of Computer Science at the University of Toronto, Toronto, ON, Canada.

Her research interests include computational linguistics, especially semantics and its relations to information theory and the theory of codes. 